\title{
Estudio de la fotocatálisis y su aplicación en el tratamiento de aguas residuales
}

\section{Photocatalysis and its application in wastewater treatment}

\author{
Sonia Esperanza Ruiz Balaguera \\ Ingeniera Química \\ Docente Universidad Nacional Abierta y a Distancia \\ sonia.ruiz@unad.edu.co
}

\section{RESUMEN}

Debido al aumento que se registra de los contaminantes recalcitrantes orgánicos en los cuerpos de aguas residuales y a las leyes ambientales regulatorias que rigen este tipo de vertimientos, muchos autores se han dedicado a desarrollar metodologías enfocadas en mitigar este impacto ambiental mediante tecnologías a base de metodologías ecológicas. Es por eso que esta revisión se centra en los procesos de oxidación como la Fotocatálisis heterogénea aplicada para tratamiento de aguas residuales ya que gracias a su acción los contaminantes orgánicos suelen ser completamente mineralizado a sustancias no tóxicas como $\mathrm{CO} 2$ y agua. Inicialmente revisamos el mecanismo de acción de la fotocatálisis es decir cómo se realiza la oxidación de contaminantes presentes en los cuerpos de agua, de igual manera abordamos los factores que de uno u otra manera tienen un efecto significativo sobre a reacción.

Palabras Clave: Foto-catalizador, Foto-mineralización, recalcitrantes, dióxido de titanio, Fotocatálisis, Fotorreactor.

\section{ABSTRACT.}

Given the increasing amounts of organic recalcitrant contaminants found in wastewater and the environmental regulations associated to its disposal, several authors have developed eco-friendly strategies to mitigate the problem. This revision aims to describe the mechanisms and factors involved in the transformation of organic residues to non-toxic compounds like $\mathrm{CO} 2$ and water by heterogenous photocatalysis and its potential application in wastewater treatment.

DOI: https://doi.org/10.22490/ECAPMA.2951 


\section{Keywords:}

Photo-catalyst,Photo-mineralization, Recalcitrants,Titaniumdioxide, Photocatalysis, Photo-reactors.

\section{INTRODUCCIÓN}

Una de las problemáticas más generalizadas que afectan la población del mundo es el inadecuado acceso al agua potable y se espera que esta problemática se vuelva critica en las próximas décadas debido a la escasez del agua en todo el mundo incluso en las zonas que se consideran ricas en este recurso.

El conocimiento a nivel mundial de la carencia del acceso al agua limpia se estima que cerca de 1.200 millones de personas carecen de agua potable, 2.6 miles de millones de personas mueren por año al no tener condiciones de sanidad y 3900 niños por día presentan enfermedades por el consumo de agua no potable. (S. Malato a, 2009) .

Los métodos convencionales de desinfección y descontaminación del agua requieren de altos requerimientos energéticos y de una gran infraestructura lo que implica una inversión considerable de capital impidiendo su implementación. Los procesos actuales para el tratamiento de aguas residuales contribuyen de cierta media al problema de contaminación ya que como resultado de estos procesos se obtienen lodos, salmueras y desechos tóxicos.

A medida que los contaminantes orgánicos recalcitrante continúan aumentando en las corrientes de agua impulsan al desarrollo de nuevas tecnologías amigables con el medio ambiente que sean capaces de mitigar este impacto ambiental.

Se ha evidenciado que la Fotocatálisis heterogénea es una herramienta eficaz en la degradación de compuestos contaminantes orgánicos presentes en estado líquido y gaseoso. Es por eso que se ha incrementado el número referencias y patentes dedicadas al estudio de la fotocatálisis ver Figura 1. 


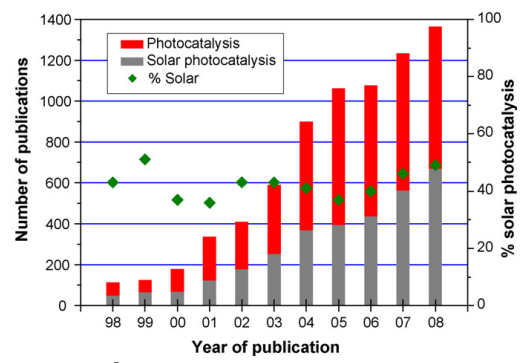

Figura 1. Breve reporte de número de publicaciones vs año de publicación.

(Malato, 2009)

Esta investigación tiene por objetivo crear una línea base de trabajo para crear proyectos con base en la fotocatálisis y su aplicabilidad en la remoción de contaminantes.

\section{METODOLOGÍA}

Inicialmente identificamos una problemática ambiental donde evidenciamos que la técnica de Fotocatálisis ha sido ampliamente estudiada para el tratamiento de aguas residuales. La revisión bibliográfica se centró en la búsqueda de artículos científicos que nos sirvieran como soporte teórico para la generación de trabajos en el marco de la remoción de agentes contaminantes presentes en las aguas residuales. Los parámetros de búsqueda empleados para la realización de este Working paper fueron en primera medida buscar si esta técnica en la actualidad es empleada para la remoción de contenientes y que tipo de contaminantes han sido re movidos por esta técnica, el cómo se realiza químicamente esta remoción (mecanismo de acción) y cuáles son los parámetros que afectan la reacción de fotocatálisis. Las revistas consultadas fueron 13 dentro de las cuales podemos destacar: Solar Energy vol 56, No 5, 401-410 (1996), Catalisis today 316, 13-20 (2018), Journal of Photochemistry and Photobiology C:Photochemistry Reviews 9, 1-12 (2008), Journal of Hazardous Materials 319, 13-23 (2016), Journal of Environmental Management 210, 328-337(2018), Catalysis Today 147 (2009) 1-59, An Quím 103, (1), 30-37 (2007), Water Research 90, 1-6 (2016), Journal of watwr Process Engineering 20, 22-28 ( 2017), Electrochimica Acta 182, 604-612 (2015), The Journal Physical chemestry Letters 4, 753-759 (2013), RSC Advance 1, 1426-1434 (2011), Chem. Soc. Rev 41, 782-796 (2012).

\section{RESULTADOS.}

\section{La fotocatálisis}

En la Figura 2 se muestra la clasificación de las diferentes tecnologías que se han implementado para el tratamiento de aguas residuales. Donde se evidencia que los procesos Fotocatalíticos degradan una gran cantidad de contaminantes orgánicos que aparecen normalmente disueltos en aguas, 
cabe resaltar que esta técnica es aplicable y/o eficiente cuando la concentración del material orgánico contaminante es baja o media como por ejemplo presencia detergentes, pesticidas y demás compuestos complejos presentes en residuos industriales, su efectividad depende también de la naturaleza del contaminante. La fotocatálisis no es viable a concentraciones superiores a 100mg* L-1 a menos que se realice un pretratamiento de dilución.

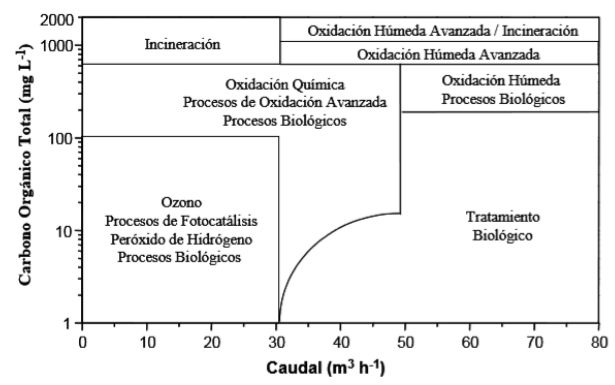

Figura 2. Diagrama de las distintas tecnologías existentes para el tratamiento de aguas en función de la carga contaminante y el volumen a tratar. (U.G.

\section{EI TiO2} Akpan, 2009)

El dióxido de titanio ( $\mathrm{TiO} 2$ ) es un conductor muy conocido e investigado, debido a la estabilidad de su estructura química y Biocompatibilidad, a sus propiedades físicas, ópticas y eléctricas. Existe en la naturaleza en cuatro formas minerales diferentes, anatasa, rutilo, brookita y titanio dióxido $\mathrm{TiO} 2$. El tipo de anatasa $\mathrm{TiO} 2$ tiene una estructura cristalina, que corresponde al sistema tetragonal dipiramidal y se utiliza principalmente como fotocatalizador bajo radiación UV. El tipo de rutilo TiO2 tiene una estructura cristalina tetragonal se utiliza principalmente como pigmento blanco, la estructura brookita tiene una estructura cristalina ortorrómbica es un mineral monoclínico y es relativamente nuevo, el titanio $\mathrm{TiO}_{2}$, es un material versátil que tiene aplicaciones en diversos productos como pigmentos de pintura, bloqueador solar, lociones, electrodos electroquímicos condensadores, células solares e incluso como agente colorante de alimentos y en pastas de dientes. (Gianluca Li Puma a, 2008)

La posible aplicación de este material como fotocatalizador en las corrientes de aguas residuales se debe a varios factores:

- La reacción fotocatalítica tiene lugar a temperatura ambiente.

- Las reacciones fotocatalíticas no sufren los inconvenientes del fotólisis debido a que no presenta la producción de productos intermedios

- Los contaminantes orgánicos suelen ser completamente mineralizado a sustancias no tóxicas como $\mathrm{CO} 2$ y agua.

- El fotocatalizador es económico y puede apoyarse en varios sustratos tales como: vidrio, fibras, acero inoxidable, materiales inorgánicos, 
arena, carbones activados, permitiendo la continua reutilizar. (U.G. Akpan, 2009)

\section{Mecanismo de acción de la fotocatálisis}

En el mecanismo de acción de la fotocatálisis se produce en primera instancia la separación de una carga debido a la excitación de un electrón (e-) desde la banda de valencia del semiconductor en el catalizador se generara así un agujero libre con carga positiva común mente llamada pares de electrón - hueco, dicha carga generada en la carga de valencia interviene en el proceso de oxidación, la carga negativa librada participa en el proceso de reducción. (Aranmedia, 2007).

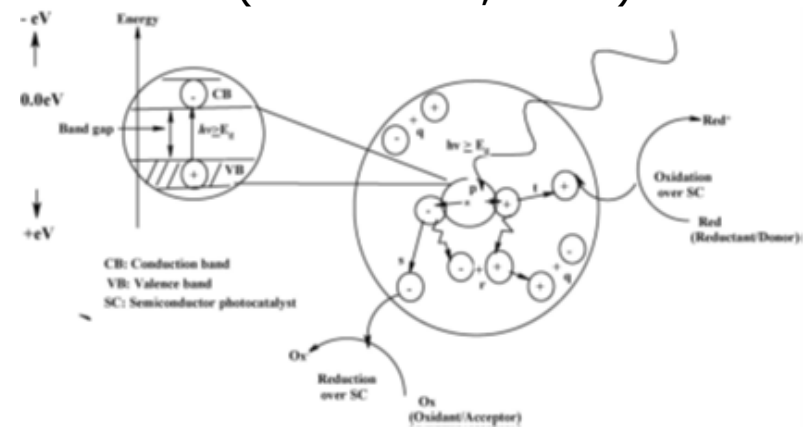

Figura 3. Diagrama explicativo del proceso de acción de la fotocatálisis empleando como catalizador TiO2. (Aranmedia, 2007)

Como producto de la reacción fotocatalítica se producen sustancias altamente reactivas con potenciales de oxidación muy altos como por ejemplo el radical hidroxilo $\left(\mathrm{Eo}=2,80 \mathrm{~V}\right.$, a $25^{\circ} \mathrm{C}$ ), que comparándolo con radicales como el cloro y el ozono que presentan potencial de oxidación 1.36 y 2.07 V respectivamente. (Quanjun Xiang, 2012)

\section{Factores que afectan la fotocatálisis}

La velocidad y eficiencia de una reacción fotocatalítica depende de un número de factores que gobiernan la cinética de la fotocatálisis tales como concentración inicial de reactivo, radiación solar UV, masa de Catalizador, $\mathrm{pH}$, temperatura, flujo radiante y concentración de oxígeno.

\section{- Concentración inicial de reactivo}

En la fotomineralización de contaminantes orgánicos sensibilizados por TiO2, se conoce tradicionalmente que la tasa inicial de desaparición del contaminante $(X)$ se ajusta a un Langmuir - Hinshelwood. La velocidad de reacción ( $r$ ) es proporcional a la fracción de superficie cubierta por el sustrato $(\theta x), \mathrm{kr}$ la constante de velocidad de reacción, C la concentración de la especie $\mathrm{X}, \mathrm{y} \mathrm{K}$ es la constante de adsorción del reactivo:

$$
r=-\frac{d C}{d t}=K r \theta \mathrm{x}=\frac{K r}{1+K C}
$$




\section{(S. Malato a, 2009)}

\section{- Radiación solar UV}

En la fotocatálisis tiene dos razones principales; primero se ajustan mejor en su rango espectral al activo. La radiación de la fotocatálisis de TiO2 a radiaciones $(\mathrm{I}<400 \mathrm{~nm})$ ampliamente disponible en el mercado o radiómetros infrarrojos para el proceso de foto-Fenton $(I<580 \mathrm{~nm})$ a escala de laboratorio con lámparas artificiales.

La intensidad del espectro solar depende de la longitud de onda; sin embargo, para caracterizar la irradiación solar o la entrada de energía en un colector solar por lo general se emplean los llamados radiómetros de banda ancha, y los espectroradiómetros.

A menudo se elige el mismo rango espectral para evaluar los resultados experimentales. Por tal razón el análisis de los resultados se simplifica considerablemente. La ecuación para medir el tiempo de irradiación normalizado es:

$$
\begin{array}{r}
t_{30 w}=t \frac{I_{G, U V}}{I_{G, U V}} \\
t_{30 w}=\frac{1}{I_{G, U V}{ }^{\circ}} \int_{0}^{t} I_{G, U V}(t) d t
\end{array}
$$

(S. Malato a, 2009)

Esta ecuación tiene que ser usada a condiciones reales, porque, como se indica, La irradiación solar nunca es constante, siendo t30 W $=0$ el momento cuando se inició la iluminación.

\section{- Masa de catalizador}

El TiO2 se utiliza a menudo como suspensión y la tasa de fotomineralización generalmente se aumenta con la concentración de catalizador la masa del catalizador depende de la geometría y condiciones de trabajo del fotorreactor dado que se encuentra diseñado para una cantidad definida de $\mathrm{TiO} 2$ en la que todas las partículas, es decir, toda la superficie expuesta, están totalmente iluminadas.

Cuando la concentración de catalizador es muy alta, la turbidez impide una mayor penetración. de luz en el reactor. Debe encontrarse una masa de catalizador óptima para evitar el exceso de catalizador y asegurar la absorción total de fotones eficientes. (García., 2015)

\section{- $\mathbf{p H}$}

El pH de la solución acuosa afecta significativamente al TiO2. La fotocatálisis en el proceso de mineralización no se ve afectada a pH entre 
valores que van desde 3 hasta 10 obteniendo porcentajes de mineralización de $80 \%$. Para trabajar fuera de estos el $\mathrm{pH}$ de las aguas residuales tendría que ser ajustado.

\section{- Temperatura}

El proceso fotocatalítico no requieren calefacción funciona a temperatura ambiente. La energía de activación suele ser muy pequeña (unos pocos $\mathrm{kJ} / \mathrm{mol}$ ) en el medio. El rango de temperatura optimo es (entre 20 y $8^{\circ} \mathrm{C}$ ). Sin embargo, a temperaturas muy bajas (por debajo de $0-8^{\circ} \mathrm{C}$ ) la energía de activación aparente aumenta. Y se limita la adsorción del producto. (Doria Argumedo C. \&., 2015)

Por otro lado, cuando la temperatura aumenta por encima de $80^{\circ} \mathrm{C}$ y acercándose al punto de ebullición del agua la adsorción de los reactivos se vuelve desfavorable la actividad disminuye y la energía de activación aparente se vuelve negativa.

Esta ausencia de calentamiento es atractiva para la reacción fotocatalítica. Ya que no se requiere perder energía para depurar aguas residuales.

\section{- Flujo radiante}

Se ha demostrado, para todo tipo de reacciones fotocatalíticas, que la velocidad de reacción es proporcional al flujo radiante. El flujo radiante se ve afectado por: la naturaleza del catalizador afectando su capacidad fotoinducida, y por ende el exceso de especies fotogeneradas ( $\mathrm{e}, \mathrm{h}+\mathrm{y}$ $\mathrm{OH}) .(\mathrm{Yu}, 2013)$

\section{- Concentración de oxígeno}

En la fotocatálisis para la purificación de aguas residuales, generalmente los contaminantes suelen ser orgánicos, por tanto, no hay fotomineralización a menos que $\mathrm{O} 2$ esté presente. Es por esto que el oxígeno se hace necesario para la completa mineralización y no parece ser competitivo con otros reactivos durante la adsorción del TiO2, ya que la oxidación tiene lugar en una ubicación diferente de donde se produce la reducción. La concentración de oxígeno también afecta la velocidad de reacción.

\section{BIBLIOGRAFÍA}

Aranmedia, A. M. (2007). Catalisis Heterogéneas y quimica Verde . An Quimica., 30-37. 
Gianluca Li Puma a, A. B. (2008). Preparation of titanium dioxide photocatalyst loaded onto activated. Journal of Hazardous Materials, 209-219.

Malato. (2009). Decontamination and disinfection of water by solar photocatalysis: . Catalysis Today, 1-59.

P. Fernandez-Ibaneza, M. M. (2009). Decontamination and disinfection of water by solar photocatalysis:. Catalysis Today, 1-59.

Quanjun Xiang, a. J. (2012). Graphene-based semiconductor photocatalysts. Chem. Soc. Rev, 782-796.

U.G. Akpan, B. H. (2009). Parameters affecting the photocatalytic degradation of dyes. Journal of Hazardous Materials, 520-529.

Yu, Q. X. (2013). Graphene-Based Photocatalysts for Hydrogen Generation. The journal Physical Chemestry Letters, 753-759.

Doria Argumedo, C., \& Deluque Viloria, H. (2015). Niveles y distribución de metales pesados en el agua de la zona de playa de Riohacha, La Guajira, Colombia. Revista de Investigación Agraria y Ambiental, 6(1), 123 - 131.

Gamboa García, D. (2015). Valoración de impactos ecológicos por minería de oro en río Guabas, Valle del Cauca, Colombia. Revista de Investigación Agraria y Ambiental, 6(2), 243 - 254. 